\title{
Macrovascular disease and systemic sclerosis
}

\author{
Meilien Ho, Douglas Veale, Clifford Eastmond, George Nuki, Jill Belch, for the East of \\ Scotland Systemic Sclerosis Study Group
}

\begin{abstract}
Objective-To determine if macrovascular disease is more prevalent in systemic sclerosis (SSc) compared with unaffected subjects.

Methods-54 patients with SSc (both limited and diffuse disease) and 43 unaffected control subjects of similar age and sex were recruited. All subjects underwent a basic screen for conventional atherosclerotic disease risk factors. All had noninvasive vascular assessments-that is, carotid duplex scanning and measurement of ankle brachial blood pressure index-to identify the presence of asymptomatic peripheral vascular disease.

Results-33 of 52 (64\%) patients had carotid artery disease compared with only 15 of $42(35 \%)$ controls $(p=0.007)$. Eleven $(21 \%)$ of these patients had moderate disease compared with only two (5\%) controls (NS). Nine of $53(17 \%)$ SSc patients had evidence of peripheral arterial disease compared with no controls. This result was also statistically significant $(p=0.003)$. There were no significant differences in the basic risk factor profile, which included cigarette smoking, systolic and diastolic blood pressure, cholesterol, trigyceride and glucose concentrations. Conclusion-Macrovascular disease is more common in SSc. Screening of these patients may allow identification of "at risk" patients at an early stage and allow the study of treatments to attenuate the high rate of cardiovascular mortality in these patients.

(Ann Rheum Dis 2000;59:39-43)
\end{abstract}

Department of

Medicine, Ninewells

Hospital and Medical

School, Dundee

DD1 9SY

$\mathrm{MHo}$

D Veale

J Belch

Department of Rheumatology, Aberdeen Royal Infirmary, Aberdeen

C Eastmond

Rheumatic Diseases Unit, Department of Medicine, The

University of

Edinburgh, Western

General Hospital,

Edinburgh

G Nuki

Correspondence to: Dr M Ho

Accepted for publication 6 September 1999 causes. We have previously reported a pilot study $^{8}$ of SSc patients, which demonstrated a $22 \%$ prevalence of symptomatic peripheral arterial obstructive disease (PAOD) as evidenced by the presence of intermittent claudi- cation detected by the Edinburgh Claudication questionnaire, which is a well validated questionnaire. This was almost five times greater than the $4.5 \%$ prevalence of symptomatic PAOD seen in the general population, ${ }^{9}$ as detected by the WHO claudication questionnaire, which is similar to the Edinburgh questionnaire. A study by Stafford et $a l^{10}$ identified excess ulnar artery disease also in SSc. If premature atherosclerosis is a feature of SSc, this would have significant implications in terms of patient treatment as it may contribute to their premature mortality. Macrovascular disease however, is not currently recognised as a feature of SSc despite its significant contribution to mortality. The aim of our study was therefore to identify the prevalence of macrovascular disease in SSc by objective laboratory testing.

\section{Methods}

PATIENTS

Fifty four consecutive patients fulfilling the 1980 American Rheumatology Association (ARA) criteria ${ }^{11}$ for SSc (diffuse cutaneous and limited cutaneous disease) were recruited from our connective tissue disease clinics. The patient pool from which these consecutive patients were taken contains the majority of the known SSc patients in the area and is unselected for vascular or any other complications. Forty three unaffected controls of similar age, sex and postal code (for area of residence) were also recruited. They were also unselected for vascular or any other complications. These controls were derived from the general population in the area who were registered with their general practioners. Any patients or controls with evidence of another connective tissue disease or rheumatoid arthritis (which may also be associated with cardiovascular disease) as shown by a positive antibody to extractable nuclear antigens (ENA) or DNA binding antibody or rheumatoid factor (RF) were excluded. Anti Scl-70 and anti-centromere antibodies were of course acceptable for SSc patients. Any patients with symptoms of Raynaud's phenomenon were excluded from being a control subject. None of the patients was receiving either intermittent or regular corticosteroid treatment nor had any significant previous history of corticosteroid treatment.

Approval for the study was obtained from the Regional Committees on Medical Research Ethics, and written, informed consent was obtained from all subjects.
BLOOD TESTS

All subjects were screened for conventional atherosclerotic risk factors, which included 
Table 1 Demographic details and risk factor profile*

\begin{tabular}{llll}
\hline & Patients & Controls & p value \\
\hline Female : male (total number) & $50: 4(54)$ & $35: 8(43)$ & \\
Age (median/range) (y) & $57(31-82)$ & $53(30-77)$ & NS \\
Diffuse: limited SSc & $13: 41$ & - & \\
Disease duration (median/range) (y) & $4.0(0.5-26)$ & - & \\
Smokers & $12(22 \%)$ & $11(26 \%)$ & NS \\
Cholesterol mean (SD) mmol/ $\dagger$ & $5.0(0.9)$ & $5.1(1.0)$ & NS \\
Number of patients with cholesterol $>5.2 \mathrm{mmol} / \mathrm{l} \dagger$ & $26(50 \%)$ & $18(48 \%)$ & $\mathrm{NS}$ \\
HDL mean (SD) $\% \dagger$ & $27.3(8.5)$ & $26.3(9.4)$ & $\mathrm{NS}$ \\
Triglycerides mean (SD) mmol/1 $\dagger$ & $1.6(0.6)$ & $1.5(0.9)$ & $\mathrm{NS}$ \\
Glucose mean (SD) mmol/1 & $5.1(1.1)$ & $5.3(1.2)$ & $\mathrm{NS}$ \\
Number of patients with glucose $>7.8 \mathrm{mmol} / \mathrm{l} \dagger$ & $2(4 \%)$ & $1(3 \%)$ & $\mathrm{NS}$ \\
Systolic BP mean (SD) mm Hg & $144(26)$ & $141(21)$ & $\mathrm{NS}$ \\
Diastolic BP mean (SD) mm Hg & $79(18)$ & $77(18)$ & $\mathrm{NS}$ \\
\hline
\end{tabular}

*None of these results was statistically different between the two groups.

†Results available for 50 patients and 37 controls.

measurement of random blood cholesterol, high density lipoprotein (HDL), triglyceride and glucose concentrations. A smoking history was obtained and blood pressure was also measured. The claudication questionnaire is not included in this paper, which reports on the laboratory findings of large vessel disease, which may be asymptomatic.

\section{NON-INVASIVE VASCULAR ASSESSMENTS}

Carotid duplex scanning and ankle brachial pressure measurements (ABPI) as measures of peripheral arterial disease, were performed on all subjects. Carotid duplex scanning (Toshiba Powervision with a $7.5 \mathrm{Mhz}$ linear probe) was performed by a vascular technician. Both common, internal and external carotid and vertebral arteries were examined for evidence of stenosis and plaque in the standard manner using a combination of B-mode ultrasound and pulsed colour Doppler signals with audio and spectral analysis. The scans were recorded on video and later assessed by a single, experienced, blinded operator. Abnormalities were recorded according to the severity of stenosisthat is, normal, minimal disease $(<20 \%$ lumen stenosis), moderate (20-49\%), severe (50$69 \%)$ and critical disease $(\geqslant 70 \%)$. Carotid plaque was classified morphologically according to B-mode appearance and based on the degree of echolucency - that is, Types I-V. ${ }^{12}$

After a 10 minute rest in a comfortably warm room, bilateral ankle and brachial arterial systolic pressures were measured using a portable Sonicaid Doppler probe and Hawkley random zero sphygmomanometer. The ABPI was calculated as the posterior tibial artery pressure in $\mathrm{mm} \mathrm{Hg}$ divided by the brachial pressure, and this was done at a later date, during the analysis of the data. The normal ABPI is 1.0 , and any value $<1.0$ is considered to be abnormal ${ }^{13}$ with the severity of the arterial disease being inversely proportional to the ABPI. ${ }^{14}$ We selected a definitive ABPI cut off point of $<$ 0.9 because this criterion has a $95 \%$ sensitivity and $100 \%$ specificity for detecting arterial disease using angiographically defined disease as the gold standard ${ }^{14}{ }^{15}$ but also evaluated the presence of milder disease with ABPI of $<1.0$, and $<0.98$.

\section{STATISTICS}

The numbers selected were calculated to enable identification of a $15 \%$ difference between patients and controls with $90 \%$ power at the 5\% significance level. Macrovascular disease data were analysed with Fisher's exact test or $\chi^{2}$ test as appropriate. Risk factors were analysed and compared using two tailed Student's $t$ test, and a p value $\leqslant 0.05$ was taken to be statistically significant. Correlations were calculated using Pearson's correlation coefficient.

\section{Results}

Demographic details for the 54 SSc patients (13 diffuse: 41 limited cutaneous) and 43 controls were similar, with no statistically significant difference between the two groups (table 1). Female:male was 50:4 for patients and 35:8 for controls.

There were no significant differences seen in the risk factors measured between patients and controls (table 1) including smoking status, mean serum cholesterol, HDL, triglyceride and glucose concentrations, and systolic and diastolic blood pressures. Similar numbers of patients and controls had an increased cholesterol of $>5.2 \mathrm{mmol} / \mathrm{l}$ (as defined by the National Cholesterol Education Program ${ }^{16}$ ). Only two patients and one control subject had an increased random glucose $(>7.8 \mathrm{mmol} / \mathrm{l}$ as defined by our laboratory's own normal range), a marker for a possible underlying diabetic state. Four patients and one control subject had a history of hypertension.

Two patients and one control did not attend for carotid scanning. Thirty three (64\%) patients had evidence of carotid disease compared with $15(35 \%)$ controls $(\mathrm{p}=0.007$, $\chi^{2}$ test) (fig 1 ). When the carotid disease was analysed according to severity, a similar trend was seen for mild disease in $22(42 \%)$ patients and $13(30 \%)$ controls and also for moderate disease, with $11(21 \%)$ patients and two (5\%) controls being affected but neither of these results was not statistically significant. Carotid plaque was present in five patients (Type IV) and three controls (Type I in one control and Type III in two controls).

One patient did not have ABPI measurements. Nine $(17 \%)$ patients had an ABPI $<0.9$ compared with no $(0 \%)$ control subjects ( $\mathrm{p}=$ 0.003 , Fisher's test). Twenty (38\%) patients and four $(10 \%)$ control subjects had an ABPI < 0.98 ( $p=0.002$, Fisher's test) and $21(40 \%)$ patients had an ABPI <1.0 compared with four $(10 \%)$ controls $(p=0.003$, Fisher's test) (fig 1$)$.

All subjects - that is, patients and controls with evidence of carotid artery disease-were evaluated independently for the presence of risk factors (table 2). The results showed that affected patients and affected controls were of similar age, with no statistically significant differences in mean serum cholesterol, HDL, triglyceride and glucose concentrations, or mean systolic or diastolic blood pressures. Two of these patients and no control subject with carotid disease were known hypertensives.

Patients with ABPI $<0.9$ had a median (range) age of 69 years (52-79) and a median (range) disease duration of 4.4 (1-10) years. They had a cholesterol mean (SD) of 5.5 (1.3) $\mathrm{mmol} / \mathrm{l}$; HDL \% of 24.9 (6.6); triglyceride 


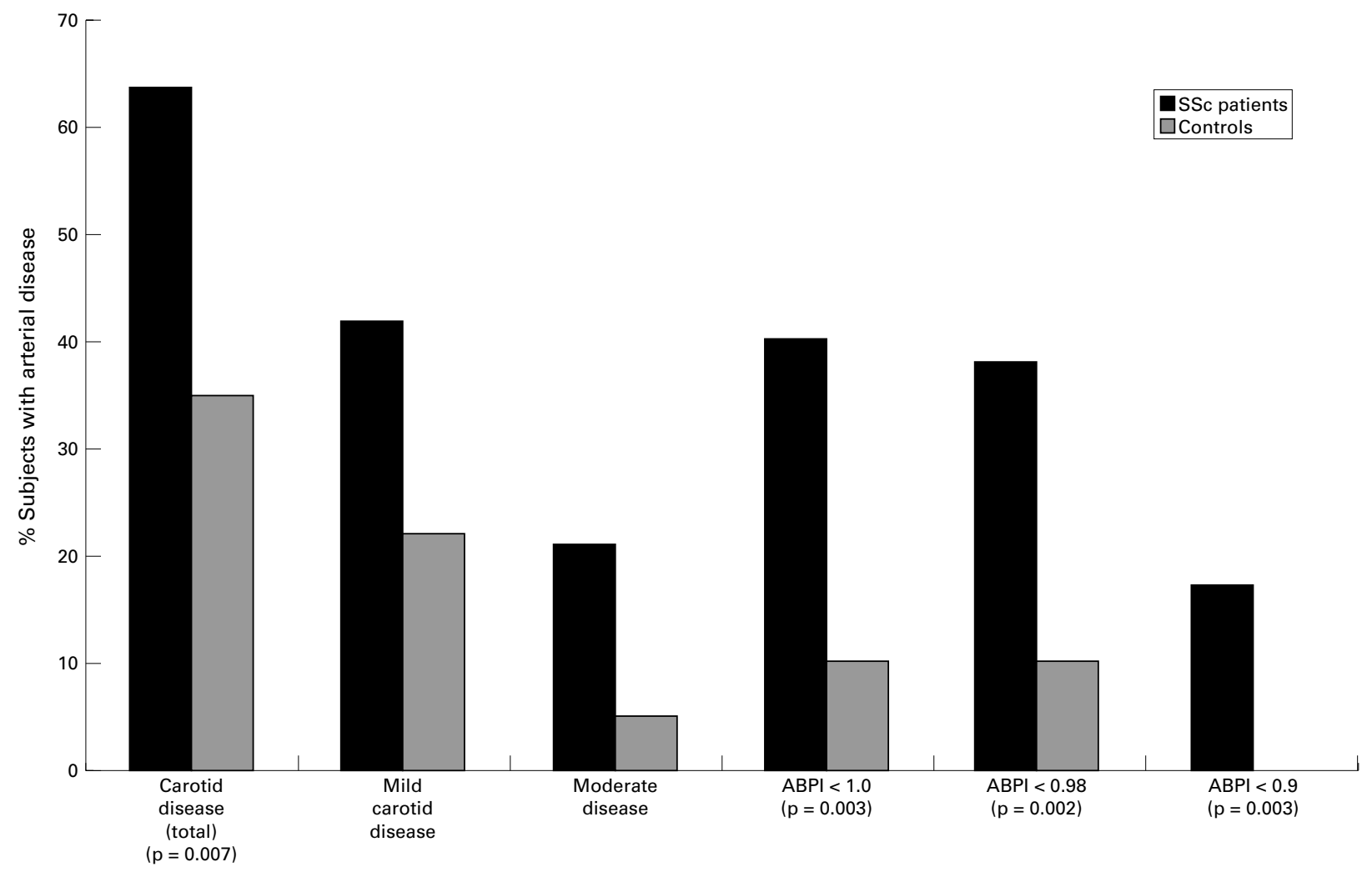

Figure 1 Prevalence of carotid and peripheral arterial disease in SSc patients compared with controls.

Table 2 Risk factor profile* of all subjects with carotid artery disease

\begin{tabular}{lll}
\hline & Patients & Controls \\
\hline Number (\%) & $33(64)$ & $15(35)$ \\
Age median (range) (y) & $61(37-82)$ & $60(45-77)$ \\
Cholesterol mean (SD) mmol/1 & $5.1(1.0)$ & $5.3(1.3)$ \\
HDL mean (SD) \% & $27.3(8.9)$ & $25.4(9.4)$ \\
Triglyceride mean (SD) mmol/1 & $1.5(0.7)$ & $1.7(1.2)$ \\
Glucose mean (SD) mmol/1 & $5.2(1.3)$ & $5.4(1.2)$ \\
Systolic BP mean (SD) mm Hg & $148(27)$ & $148(18)$ \\
Diastolic BP mean (SD) mm Hg & $77(19)$ & $84(9)$ \\
\hline
\end{tabular}

^Not statistically significant.

mean (SD) of $1.7(0.6)$ and glucose mean (SD) of 5.4 (1.9). Mean systolic and diastolic blood pressures were $156(26) / 85(7) \mathrm{mm} \mathrm{Hg}$. One patient had a history of hypertension. There were no controls with an ABPI $<0.9$.

History taking showed that three patients had a history of peripheral arterial disease and three a history of cerebrovascular disease while no controls had a history of peripheral arterial disease or cerebrovascular disease. There was no correlation between macrovascular disease and disease duration or SSc subset (Pearson's correlation coeficient).

\section{Discussion}

Our results show, for the first time by standard laboratory testing, that patients with SSc have an increased prevalence of macrovascular disease in the form of carotid artery disease and PAOD. In this study, carotid disease has been assessed in the same way as for atherosclerotic carotid disease and findings include the detection of plaques characteristically seen in conventional atherosclerotic disease, as well as vessel stenoses. The role of duplex scanning in the investigation of carotid artery disease is well established, and there is evidence to suggest that it has a predictive role in identifying those patients with a greater than normal risk of stroke. ${ }^{17-19}$ Our patients seem to have more severe, as well as more frequent, carotid disease, and such duplex findings suggest that these SSc patients may have an increased risk of a stroke. There is however, no scientific evidence at present to indicate an excessive occurrence of stroke disease in these patients. Our findings, using well validated techniques, contrast with that of a previous study ${ }^{10}$ showing no excess of carotid or lower limb arterial disease in SSc. There were, however, only 20 patients in this study, which also used a different methodology for measurement of intraluminal diameter and limited the examination to specific sites.

ABPIs are routinely used in the investigation of atherosclerotic PAOD. Two of our SSc patients subsequently developed critical limb ischaemia and required distal grafting. Pathology samples showed a degenerative process indistinguishable from conventional atheroma. Neither of these female patients aged 49 and 50 years was diabetic nor had an increased cholesterol. One was a smoker. In our study, the basic conventional atherosclerotic risk factor profile in our SSc patients is indistinguishable from that of unaffected control subjects. If anything, the male to female ratio was slightly higher in the control group and this might be expected to result in a bias in favour of the SSc group as being female offers some protection against atherosclerotic vascular disease. The selection of the control group is another point for 
discussion. We recognise that the usual method is to select alphabetically matched controls from general practice lists but felt it would be more appropriate to match the subjects for social class as social habits and behaviour are important factors in cardiovascular morbidity and mortality. Thus we selected age and sex matched controls with matching for postcode areas.

There are similarities in clinical symptomatology between SSc and atherosclerotic disease with digital and lower limb ulcers occurring in both clinical scenario. Further similarities are seen in the histopathology of the affected vessels ${ }^{20-23}$ and the aetiopathologenesis of both diseases. ${ }^{24-28}$ The question therefore, is whether this macrovascular disease of SSc is atherosclerotic in nature: this remains unanswered at present.

Nevertheless, this finding has important clinical implications and targeted treatment may be indicated in high risk patients. PAOD itself is associated with increased cardiovascular mortality. The degree of lowering of the ABPI is correlated to the severity of disease ${ }^{14}$; disease severity is, in turn, directly correlated with an increasing risk of both fatal and non-fatal cardiovascular events at all levels of a reduced ABPI from $\leqslant 0.7$ up to $1.0 .^{29} \mathrm{In}$ women, an ABPI < 0.9 confers a 2.1 (95\% CI $1.4,3.1)$ relative risk of cardiovacular mortality $^{30}$ and with an ABPI $<0.85$, the relative risk for cardiovascular events is 4.0 (1.3, $8.5) .{ }^{31} \mathrm{SSc}$ is also associated with a higher rate of cardiovascular mortality compared with unaffected persons. ${ }^{8}$ This is not universally appreciated; mortality studies generally classify deaths as being either related to or unrelated to SSc and many cardiovascular deaths are automatically (but incorrectly we believe) assumed to be unrelated to SSc and categorised as a non-SSc related death. Support for this belief comes from a recent study, ${ }^{32}$ which showed that SSc patients experienced twice the expected number of non-SSc related deaths, the majority of which were cardiovascular in nature. The overall pattern of increased large vessel disease associated with cardiovascular events mirrors that of atherosclerotic disease. Large studies have shown a significant decrease in cardiovascular events in patients with atherosclerotic peripheral arterial disease treated with aspirin and other antiplatelet agents. ${ }^{33}{ }^{34}$ Aspirin however may be poorly tolerated in SSc patients because of oesophageal abnormalities ${ }^{35}$ and should only be used if the benefits outweigh the risk from side effects. Perhaps other anti-platelet agents such as clopidogrel ${ }^{36}$ should be evaluated, but the rationale for the use of other more expensive compounds needs to be validated.

In summary, we have shown an increased prevalence of macrovascular disease in SSc. Further work is required in this area to elucidate the nature of the macrovascular abnormalities and the risk factors associated with it, which may well be amenable to treatment and allow attenuation of the high rate of cardiovascular deaths in this group of patients.
Funding: this study was funded by the Raynaud's and Scleroderma Association, UK. The duplex scanning equipment was purchased through a grant from the Medical Research Council.

1 Belch JJF, Zoma A, Richards, Forbes CD, Sturrock RD. Vascular Damage and factor VIII related antigen in the rheumatoid diseases. Rheumatol Int 1987;7:107-11.

2 Larkin JG, Belch JJF, Flannigan P, Forbes CD, Frier BM. Microvascular disease and limited joint mobility in diabetes. A comparision of fibrinolysis and and prostacycdiabetes. A comparision of fibrinolysis and and prostacyc-
lin in diabetes and systemic sclerosis.Diabetic Med 1988;5: 53-6.

3 Lau CS, McLaren M, Belch JJF. Factor VIII von Willebrand factor antigen levels correlate with symptom severity in patients with Raynaud's phenomenon. Br J Rheumatol 1991;30:433-6.

4 Lau CS, McLaren M, Mackay I, Belch JJF. Baseline plasma fibinolysis and its correlation with clinical manifestations in patients with Raynaud's Phenomenon Ann Rheum Dis 1993;52:443-8

5 Lau CS, O'Dowd A, Belch JJF. White blood cell activation in Raynaud's phenomenon of systemic sclerosis and VWF syndrome. Ann Rheum Dis 1992;51:249-52.

6 Galt SW, McDaniel MD, Ault KA, Mitchell J, Cronenwett JL. Flow cytometric assessment of platelet function in patients with peripheral arterial occlussive disease. J Vasc Surg 1991;14:747-55.

7 Bryan C, Howard Y, Brennan P, Black C, Silman A. Survival following the onset of scleroderma: results from a retrospective inception cohort study of the UK patient retrospective inception cohort study of the
population. Br J Rheumatol 1996;35:1122-6.

8 Veale DJ, Collidge TA, Belch JJF. Increased prevalence of Veale DJ, Collidge TA, Belch JJF. Increased prevalence of
symptomatic macrovascular disease in systemic sclerosis. Ann Rheum Dis 1995;54:853-5.

9 Fowkes FGR, Housley E, Cawood EHH, MacIntyre CCA, Ruckley CV, Prescott RJ. Edinburgh Artery Study: Prevalence of asymptomatic and symptomatic peripheral arterial disease in the general population. Int J Epidemiol 1991;20:384-92.

10 Stafford L, Englert H, Gover J, Bertouch J. Distribution of macrovascular disease in scleroderma. Ann Rheum Dis 1998;57:476-9.

11 Subcommittee for Scleroderma Criteria of the American Rheumatism Association Diagnostic and Therapeutic Criteria Committee: Preliminary criteria for the classification of systemic sclerosis (scleroderma). Arthritis Rheum 1980; 23:581-90.

12 Faris I, McCollum P, Mantese V, Lusby R. Investigation of the patient with atheroma. In: Bell PRS, Jamieson CW, Ruckley CV, eds. Surgical management

13 Walker WF, Spence VA and McCollum PT. Systolic pressure measurements in the ischaemic lower limb. Hospital Update 1986:343-58.

14 McKenna M, Wolfson S, Kuller L. The ratio of ankle and arm arterial pressure as an independent predictor of mortality. Atherosclerosis 1991;87:118-28.

15 Bernstein EF, Fronek A. Current status of non-invasive tests in the diagnosis of peripheral arterial disease. Surg Clin North Am 1982;62:473-87.

16 National Cholesterol Education Program. Detection, evaluation and treatment of high blood cholesterol in adults (Adult Treatment Panel II). National Institutes of Health. NIH Pub 1993;93:3095.

17 Roederer GO, Langlois YE, Lusiani L, Jager KA, Primozich JF, Lawrence RJ, et al. The natural history carotid arterial JF, Lawrence RJ, et al. The natural history carotid arterial disease in asymptomatic

18 Johnson BF, Verlato F, Bergelin RO, Primozich JF, Strandness E Jr. Clinical outcome in patients with mild to moderate carotid artery stenosis. J Vasc Surg 1995;21:120-6.

19 Laing S, Greenhalgh RM. The detection and progression of asymptomatic peripheral arterial disease. Br J Surg 1983;70:628-30.

20 D'Angelo W, Fries JF, Masi AT, Schulman LE. Pathologic observations in sysyemic sclerosis (scleroderma).Am J Med 1969;46:428-39.

21 Leinwald L, Duryea AW, Richter MN. Scleroderma (based on a study of over 150 cases). Ann Intern Med 1954;41:1003.

22 Goetz RH. The pathology of progressive systemic sclerosis(generalised scleroderma) J Capetown Postgrad Med Ass 1945;4:337-02.

23 Antonovych TJ, Steingaszner LC. Pathologic changes in progressive systemic sclerosis. Georgetown Med Bull 1962;16:27.

24 Ross R.The pathogenesis of atherosclerosis:a perspective for the 1990s. Nature 1993;362:801-9.

25 Masuda J, Ross R. Atherogenesis during low level hypercholesterolaemia in the non-human primate.I. Fatty streak formation. Arteriosclerosis 1990;10:164-77.

26 Masuda J, Ross R. Atherogenesis during low level hypercholesterolaemia in the nonhuman primate.II Fatty streak conversion to fibrous plaque. Arteriosclerosis 1990;10: 178-87.

27 Belch JJF. The white blood cell as a risk factor for thrombotic vascular disease. Vascular Medicine Review 1990;1:203-13.

28 Bruckdorfer R, Hillary JB, Bunce T, Vancheeswaran R, Black CM. Increased susceptibility to oxidation of low density lipoproteins isolated from patients with systemic density lipoproteins isolated from patients
sclerosis. Arthritis Rheum 1995;38:1060-7. 
29 Leng GC, Fowkes FGR, Lee AJ, Dunbar J, Ruckley CV. Use of ankle-brachial pressure index to predict cardiovascular 30 Vogt MT, McKenna M, Anderson SJ, Wolfson SK, Kuller $\mathrm{LH}$. The relationship between ankle-arm index and mortality in older men and women. J Am Geriatr Soc 1993;41:523-30.

31 Vogt MT, Cauley JA, Kuller LH, Hulley SB. Decreased ankle/arm blood pressure index and mortality in women JAMA 1993;240:465-9.

32 Jacobsen S, Halberg P, Ullman S. Mortality and causes of death of 344 Danish patients with systemic sclerosis (scleroderma). Br J Rheumatol 1998;37:750-5.
33 Duprez D, Clement DL. Medical treatment of peripheral vascular disease: good or bad? Eur Heart J 1992;13:14951

34 APT Collaboration, Clinical Trial Service Unit, Nuffield Department of Clinical Medicine, Radcliffe Infirmary, Oxford. BMJ 1988;296:320-31

35 Torres MA, Furst DE. Treatment of systemic sclerosis. Rheum Dis Clin North Am 1990;16:217-41.

36 CAPRIE Steering Committee. A randomised, blinded trial of clopidogrel versus aspirin in patients at risk of ischaemic events (CAPRIE). Lancet 1996;348:1329-39. 